\title{
Unsteady Numerical Investigation on Vortex Interaction between Rim Seal Purge Flow and Upstream Stator
}

\author{
L. Zhou, Z. X. Wang ${ }^{\dagger}$, F. Yang and J. W. Shi \\ School of Power and Energy, Northwestern Polytechnical University, Xi'an, Shaanxi Province, China \\ †Corresponding Author Email: wangzx@nwpu.edu.cn
}

(Received February 6, 2021; accepted September 23, 2021)

\begin{abstract}
To assess unsteady vortex interaction between rim seal purge flow and upstream stator, numerical investigations were conducted under different purge flow rates. The vortex distributions for the stator and cavity were investigated and the interaction processes near the cavity exit, in particular the vorticity change resulting from the ingress and egress, were analyzed. Results show the intensity of hub passage vortex (HPV) and hub trailing shedding vortex (HTSV) at stator exit is decreased as a consequence of enhancing blockage effects caused by the egress flow. However, when the purge flow rate increases, from stator exit to downstream of cavity exit, the reduction in the intensity of two vortices is weakened as the extrusion of egress flow thins their vortex tubes. The vortex inside the cavity is generated as the combined effect of relative rotation of cavity walls and nonuniform circumferential pressure mainly imposed by upstream stator. The ingress leads the positive axial vorticity near the stator hub to ingest into the cavity and eject into the main passage due to the blockage of purge flow. Furthermore, the interaction between the ingress of the mainstream and purge flow produces local negative axial vorticity. The egress flow carries negative axial vorticity mainly originated from the rotational cavity wall, and enters into the main flow passage near the rotating hub, then locations of HPV and HTSV move to the mid-span slightly with the extrusion of egress flow.
\end{abstract}

Keywords: Rim seal; Ingress; Egress; Stator; Vortex.

\section{NOMENCLATURE}

$$
\begin{array}{ll}
C_{p s} & \text { pressure coefficient, } \\
& \mathrm{C}_{p s}=\left(P_{s}-P_{\mathrm{s}, 2}\right) /\left(P_{t, 1}-P_{\mathrm{s}, 2}\right) \\
\text { NAL } & \text { normalized axial location }
\end{array}
$$
$P_{S}$ local static pressure
$P_{s, 2}$
$P_{t, 1}$

\section{INTRODUCTION}

When the gas temperature before turbine has been improved constantly, the overheating of the discs and thermal fatigue phenomenon is becoming more and more obvious, great attention has been concerned on the ingress of turbine main flow gas into the disc cavity. To reduce the hot turbine main flow ingestion, the rim seal is arranged and the rim seal purge flow is adopted to cool the disc cavity. Owing to the high pressure of the rim seal purge flow, it ejects into the main flow passage and has impact on the main flow, inducing flow loss (Schobeiri et al. 2015; Jia et al. 2018, Zhu et al. 2014).

The rim seal purge flow is generally found to reduce the rotor blade loading and increase the secondary flow loss, then the turbine performance becomes worse (Turgut and Camc1 2012; Cui and Tucker
2016; Hu et al. 2017). Thus, it is necessary to study the interaction between the purge flow and the mainstream, and clarify the mechanism of interaction and flow loss.

The purge flow introduces an additional vortex structure due to the impact of the purge flow on mainstream (Gier et al. 2003; Schlienger et al. 2003; Blanco et al. 2006). Popović and Hodson (2010) showed that the leakage slot vortex was originated from the outer part of rim seals and had impact on the interaction between the purge flow and mainstream as it gradually developed as the HPV. A similar vortex system, labeled as junction vortex, was described by Chilla et al. (2012). This vortex resulted from the ingested mainstream merging with the front fin separation vortex and occupied the main part of the front fin. Jia and Liu (2013, 2014) conducted numerical investigations of the interaction 
between purge flow of the upstream cavity and mainstream, and found two vortex structures, which were named as the slot leakage vortex and shear induced vortex. The shear induced vortex was generated as the difference in circumferential momentum and developed into the hub passage vortex. This vortex was similar to the vortex system proposed by Popović and Hodson (2010) and Chilla et al. (2012). Schupbach et al. (2011) also found two additional vortices like Jia and Liu (2013, 2014) when there was purge flow, and stated that a streamwise vorticity component was created when additional vortex was rounded the rotor leading edge. Therefore, the purge flow caused additional vortex in the main passage and interacted with the hub secondary flow of downstream rotor. However, part of the inducing vortices in the main passage was originated from the vortex in the cavity. Thus, the vortex in the cavity, especially near the cavity exit, requires further study.

Furthermore, the purge flow interacted with the secondary flow of the mainstream (Gibson 2011; Wright et al. 2007). Gallier and Lawless (2004) showed that the negative vorticity induced by the wake suppressed the purge flow along radial direction and promoted reattachment of the boundary layer. Gao et al. (2007) reported the upstream hub passage vortex was unfavorable for the purge flow to cool the mainstream, and the film-cooling effectiveness of the platform was decreased correspondingly. Schadler et al. (2016) stated the purge flow was under the stator hub passage vortex and trailing shedding vortex, which implied that these two vortices were benefit to the intrusion of the purge flow.

Therefore, a strong interaction existed between the purge flow and upstream secondary flow, and the detailed interaction should be concerned to obtain the influence of rim seal purge flow on upstream stator.

From the description of the above research, it showed the purge flow created additional vortex structures and interacted with secondary flow of the stator. However, the induced additional vortices in the main passage may be originated from the vortex inside the cavity, the development of the vortex structure inside the cavity is lack of focus. Furthermore, the effect of ingress and egress related to the purge flow on the vortex interaction is unknown, and the detailed vortex interaction process between upstream stator secondary flow and rim seal purge flow, appearing around the cavity exit is still a question, it is of great significance to obtain the relationship between the vortex and ingress / egress flow phenomena. Therefore, the unsteady vortex interaction between rim seal purge flow and upstream stator is investigated.

The vortex distribution for the stator and cavity is analyzed, and the interaction process upstream, downstream and above of cavity exit is discussed, in particular vorticity changes resulting from the ingress and egress. Then, the interaction mechanism between rim seal purge flow and upstream stator is explored.

\section{COMPUTATIONAL SCHEMES}

\subsection{Numerical method and calculation model}

ANSYS CFX was chosen to perform numerical simulations, unsteady Reynolds-averaged NavierStokes equations was solved, with SST $k-\omega$ turbulence model adopted. The time discretization was realized by the Euler scheme with second-order backward difference, and second-order upwind scheme for spatial discretization.

1.5 axial turbine stage "LISA" with an axial rim seal was simulated. The detailed geometric structures of the turbine and the rim seal cavity can be found in the investigation of Behr (2007) and Schupbach (2009), and the configuration of calculation model is given in Fig. 1.

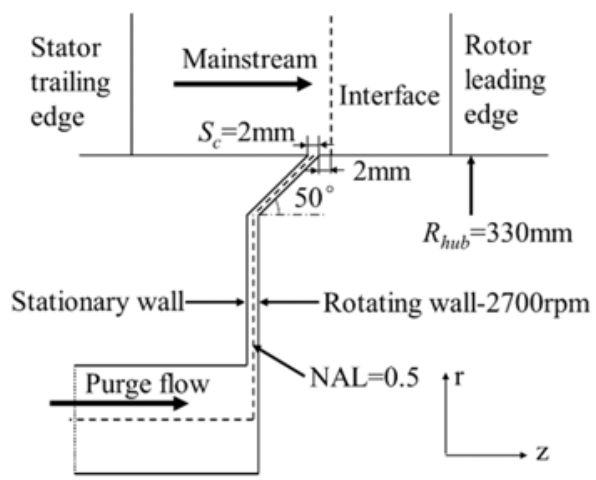

Fig. 1. Configuration of calculation model.

As displayed in Fig.1, the interface was arranged downstream of the cavity which is chosen according to the investigation of Schupbach (2009), the stator vane and cavity belong to the stationary calculation domain, and the rotor blade was included in the rotating zone. For the flow in the rotating zone, the flow field was solved in the relative frame.

The structured mesh of calculation model was presented in Fig. 2. The number of stators and rotors are 36,36 and 54 , the unsteady calculation is performed in two stator channels and three rotor channels. The total number of 7.83 million was selected and validated using grid independence analysis, of which 7.29 million and 0.54 million were adopted for turbine main passage and the cavity respectively. The thickness of the first layer grid to the wall was specified as $0.001 \mathrm{~mm}$ to satisfy the $y^{+}$ requirement of the SST $k-\omega$ turbulence model.

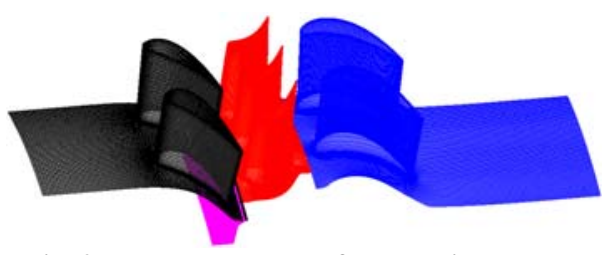

Fig. 2. structured mesh of calculation model. 


\subsection{Boundary conditions}

The total temperature, total pressure and flow angle were prescribed at the first stator inlet. Pressure outlet boundary was imposed at the second stator outlet with static pressure profile described (Behr 2007). At the cavity inlet, the mass flow rate was employed. The boundary of cavity wall near the stator hub was set as stationary wall, and the cavity wall close to the rotor hub was specified as rotating wall. The mass flow rate ratio of the rim seal purge flow at the cavity inlet to the mainstream was defined as injection ratio (IR). At the mainstream inlet, the turbulence intensity was set as $10 \%$, and $5 \%$ was specified at the cavity inlet according to the investigation of Schupbach (2009). The detailed boundary condition parameters were given in Table 1.

Table 1 Computation boundary conditions.

\begin{tabular}{|c|c|}
\hline Boundary condition & Value \\
\hline Total pressure of mainstream $/ \mathrm{Pa}$ & 140000 \\
\hline $\begin{array}{c}\text { Total temperature of } \\
\text { mainstream } / K\end{array}$ & 328.15 \\
\hline Rotational speed $/ r p m$ & 2700 \\
\hline $\begin{array}{c}\text { Total temperature of rim seal } \\
\text { purge flow } / K\end{array}$ & 323.15 \\
\hline IR/\% & $0.5,0.9,1.3$ \\
\hline \multicolumn{2}{|c|}{} \\
\hline
\end{tabular}

\subsection{Validation of numerical method}

Calculation was performed and compared with experimental results (Schuepbach 2009, 2011) to verify the numerical method adopted. The unsteady flow field was measured by a fast response aerodynamic probe, with $0.8 \%$ the relative measurement uncertainties for yaw angle and $1 \%$ for total pressure (Schuepbach et al. 2011). Figure 3 displays the comparison between the simulation and experimental data at the rotor exit, where IR is $0.9 \%$. It can be found the predicted relative total pressure and flow yaw angle are consistent with the experimental result of Schuepbach et al. (2011). The maximum deviation between the calculation and the experimental data does not exceed $3.5 \%$ except for the region near the tip. Therefore, the numerical method adopted can be used to investigate the interaction between the rim seal purge flow and mainstream.

\section{RESULTS AND DISCUSSION}

\subsection{Upstream of the cavity exit}

To directly display the vortex distributions near the cavity exit, contours of the time-averaged axial vorticity for stator and cavity cutting planes are given in Fig. 4. The circumferential region is divided into fifteen parts, where the interval of each part is 1.333 degree. As a result, the unsteady and non-uniform distributions of axial vorticity can be observed clearly. Every cutting plane is labeled by

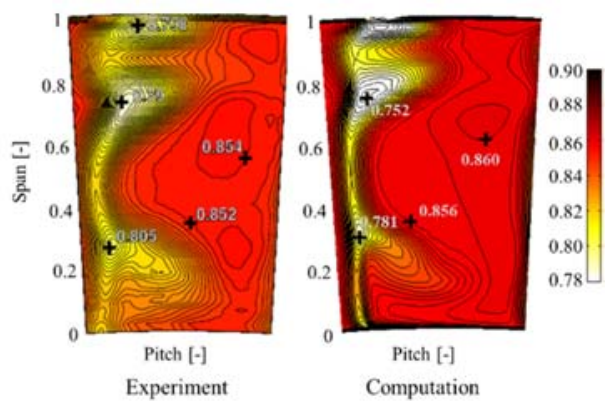

(a) Relative total pressure coefficient contours

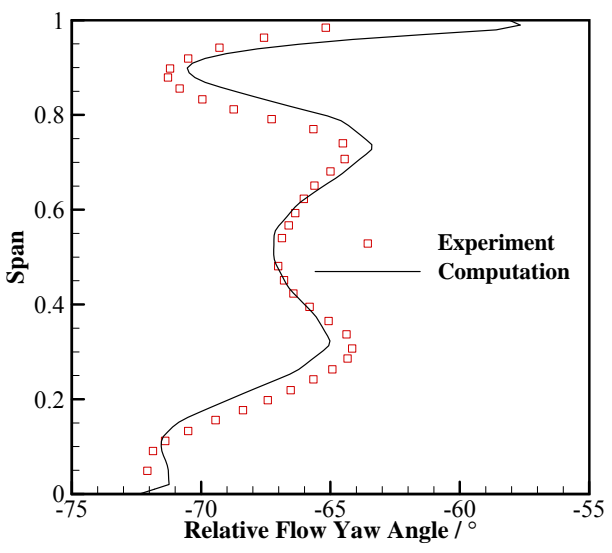

(b) Relative flow yaw angle

Fig. 3. Comparison between the simulation and experimental data $(\mathrm{IR}=\mathbf{0 . 9 \%})$.

corresponding numbers, among them planes 7 is located downstream of stator trailing edge.

When IR is $0.5 \%$, inside the cavity, significant negative axial vorticity A appears downstream of stator trailing edge (labeled by the rectangle), especially at the cutting plane of 7 (shown in the zoomed view). In the mainstream passage, the positive axial vorticity $B$ nearly covers the cavity exit at cutting plane 7 and the negative axial vorticity $\mathrm{C}$ exists near the rotating hub. Under IR of $0.9 \%$, the negative axial vorticity $A$ inside the cavity decreases significantly and the positive axial vorticity B and negative axial vorticity $\mathrm{C}$ in the main passage strengthen. For $\mathrm{IR}=1.3 \%$, the negative axial vorticity A disappears and the positive axial vorticity $\mathrm{B}$ is pushed to higher radial position and positive axial vorticity $B$ and negative axial vorticity $C$ become more significant. Furthermore, downstream of stator suction side, the radial location of positive axial vorticity $B$ increases compared with that downstream of stator trailing edge, which can be found in the zoomed view for $\mathrm{IR}=1.3 \%$.

To investigate the cause of axial vorticity distributions in Fig. 4, radial velocity distribution at the cavity exit is presented in Fig. 5 under different IR. The radial velocity can be used to judge the phenomenon of the mainstream ingestion and the rim seal flow ejection. When the radial velocity is negative, it means the flow direction is downward and the mainstream is pushed into the cavity, 


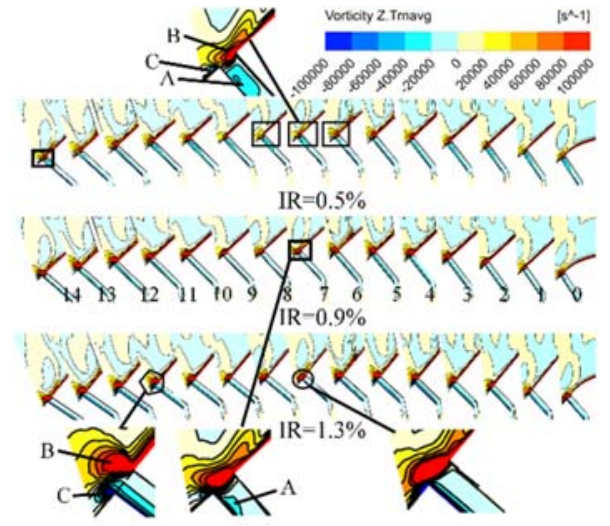

Fig. 4. Time-averaged axial vorticity distribution for cutting planes.

meaning mainstream ingestion. On the contary, the radial velocity with positive value denotes the rim seal purge flow ejecting into the mainstream. Under diffferent IR, it can be seen that the the mainstream ingestion occurs near planes 7 , which is downstream of stator trailing edge, and the rim seal purge flow egress occurs downstream of stator suction side. The ingress and egress phenomenon are induced by the pressure difference between the mainstream and the cavity. Due to the high pressure downstream of stator trailing edge, which is higher than the pressure in the cavity, a positive pressure gradient is generated at the cavity exist, and then the mainstream ingress is formed. Similarly, the purge flow egress appears owing to the negative pressure difference, as the pressure downstream of stator suction is lower than that it has in the cavity. As shown in Fig. 5, the strong mainstream ingress occurs for IR of $0.5 \%$, leading to the negative axial vorticity A (labeled in Fig. 4). With the increment of IR, the mainstream ingress becomes weaker because the pressure in the cavity increases under larger IR, which reduces the positive pressure gradient between the mainstream and the cavity.

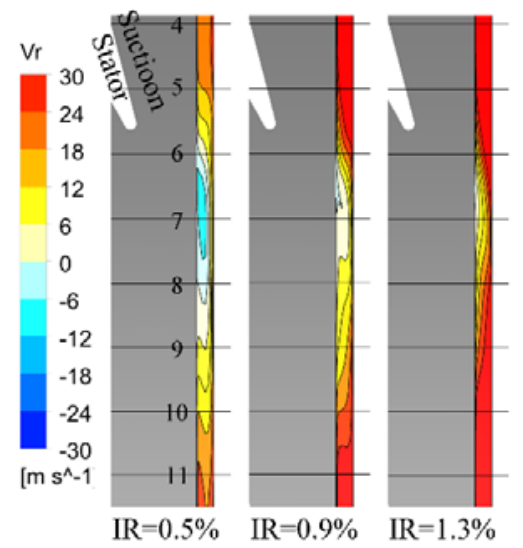

Fig. 5. Time-averaged radial velocity distribution at cavity exit.

Therefore, the zone of the mainstream ingress decrease, and the zone of purge flow egress enlarges correspondingly with the increase of IR. Under IR of $1.3 \%$, the mainstream ingress nearly disappears, the purge flow egress occupies the whole cavity exit., then the rim seal purge flow gets into turbine passage and interacts with the mainstream as shown in Fig. 6, the egress flow causes intensifying of the positive axial vorticity $B$ and negative axial vorticity $C$ (labeled in Fig. 4) in the main passage.

In order to observe the stator passage vortex, the schematic figure of the vortex types in the stator passage and time-averaged axial vorticity distribution at upstream stator exit are displayed in Fig. 6. The location of stator exit is labeled in Fig. 5 and the span positions are labeled with corresponding numbers. The HPV is the zone of negative axial vorticity and is mainly located from the hub to $10 \%$ span. HTSV is the zone of positive axial vorticity can be found between the $10 \%$ span and $20 \%$ span. The HTSV is formed owing to the interaction between the suction-side boundary layer and HPV. From the case without the cavity to IR = $1.3 \%$, the radial locations of HPV and HSTV increase slightly. Furthermore, the intensity and size of HPV and HTSV reduce with the increment of purge flow rate.

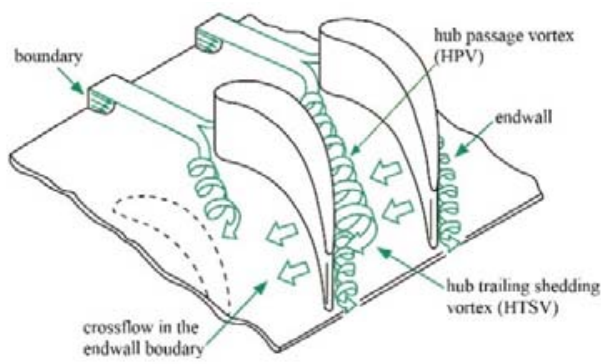

(a) schematic figure of the vortices types

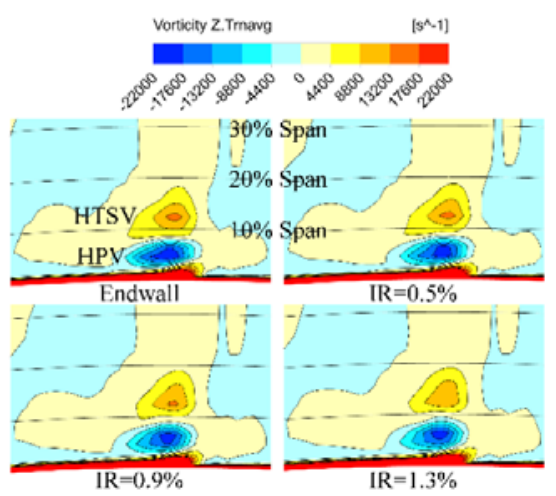

(b) axial vorticity at stator exit

Fig. 6. Time-averaged axial vorticity distribution at stator exit.

The pressure coefficient at $5 \%$ span of the stator is presented in Fig. 7. The pressure coefficient near the stator suction side continues to improve when IR is increased. This is because the egress flow is mainly located downstream of stator suction side and correspondingly introduces blockage to the stator suction side. This leads to the increase in the local 


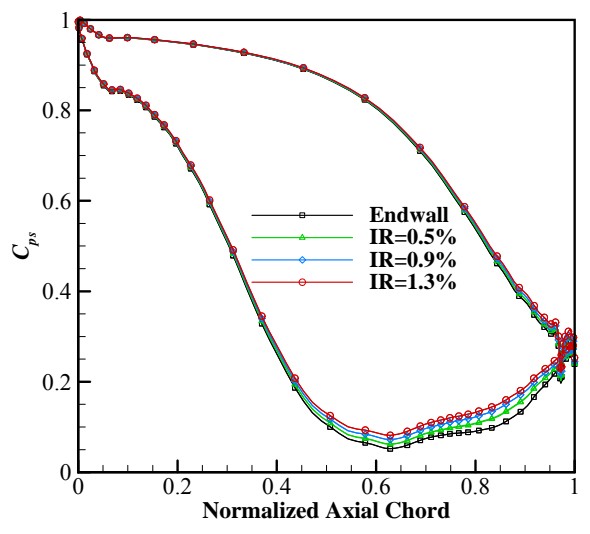

Fig. 7. Pressure coefficient at $5 \%$ span of the stator.

pressure which can be seen in Fig. 5. Thus, the pressure gradient along the pitch wise direction reduces and the intensity of hub secondary flow decreases. Furthermore, the egress flow is emanated from the hub and causes significant blockage near the hub as shown in Fig. 5. This results in the pressure gradient towards the casing and the radial migration of the HPV and HTSV.

To summarize, the mainstream ingress becomes weaker with the increment of IR, resulting in a decrease of the negative axial vorticity A inside the cavity, and egress becomes stronger, leading to an increase of the positive axial vorticity $\mathrm{B}$ and negative axial vorticity $\mathrm{C}$ in the main flow passage. In addition, the strengthening egress causes significant blockage to the suction side, which contributes to the reduction of the size and intensity of HPV and HTSV.

\subsection{Inside the cavity and at the cavity exit}

To investigate the axial vorticity distributions inside the cavity, the axial vorticity contours at two axial planes, namely near the stationary wall $(\mathrm{NAL}=0.05)$ and near the rotating wall (NAL $=0.95)$ are given in Fig. 8. NAL represents the normalized axial location, which starts from the stationary wall of the cavity to the rotating wall of the cavity. The location of NAL $=0.5$ has been labeled in Fig. 1. The circumferential region is also divided into fifteen parts and corresponding circumferential locations are labeled. The two axial planes stretch from the rim seal inlet to $10 \%$ span in the main passage. The location of the HPV is labeled, which corresponds to the distributions in Fig. 6.

At $\mathrm{NAL}=0.05$, the negative axial vorticity, corresponding to the negative axial vorticity A connected with the ingress in Fig. 4, completely covers the circumferential range under IR of $0.5 \%$. From IR $=0.5 \%$ to IR $=1.3 \%$, the negative axial vorticity greatly reduces (as shown the arrow b). In addition, the negative axial vorticity for IR $=0.5 \%$ changes to positive axial vorticity for IR $=0.9 \%$ and $\mathrm{IR}=1.3 \%$ (as shown the arrow a) and positive axial vorticity gradually increases from $\mathrm{IR}=0.9 \%$ to $\mathrm{IR}=$ $1.3 \%$. It can be seen that the interface between the positive and negative axial vorticity is just under the
HPV. This implies the purge flow encounters the high-pressure region downstream of the stator trailing edge and divides into two parts. One part is towards the right direction (downstream of stator pressure side) and forms the negative axial vorticity. Another part is towards the left direction (downstream of the stator suction side) and develops into the positive axial vorticity. With the increment of IR, more egress is occurred and more purge flow enters into the main flow passage which is downstream of stator suction side. Thus, the region of negative axial vorticity decreases in size while the region of positive axial vorticity increases. Furthermore, the intensity of the negative axial vorticity reduces. This results from the decrease of pressure gradient towards the cavity under larger IR.

At NAL $=0.95$, negative axial vorticity completely covers the circumferential range from IR $=0.5 \%$ to $\mathrm{IR}=1.3 \%$. The circumferential velocity of the purge flow near the rotating wall reaches the rotor hub circumferential velocity while that near the stationary wall becomes 0 due to the relative rotation of cavity walls. As a result, the negative axial vorticity is developed inside the cavity, in particular near the rotating wall.

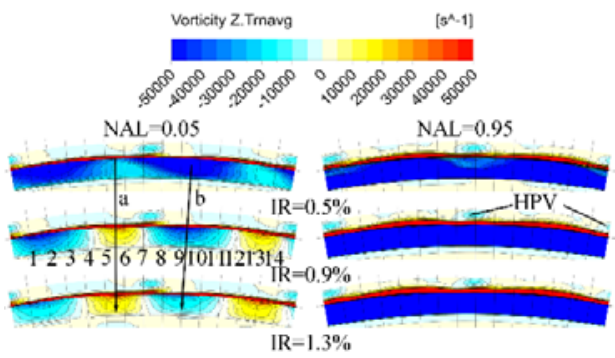

Fig. 8. Axial vorticity contours for three axial planes.

To investigate the unsteady behavior of vortices, in particular the vorticity changes related to the ingress, time-resolved and time-averaged axial vorticity contours at cutting planes 7 and 11 for $\mathrm{IR}=0.5 \%$ are given in Fig. 9. The corresponding surface streamlines are added to the plane inside the cavity to reveal the influence of ingress on the axial vorticity distributions. The anti-clockwise vortices are labeled by blue arrows and the Tr represents the rotor blade passing period.

At cutting plane 7 , the positive axial vorticity enters into the cavity at three time-instances except the $1 / 5$ $\operatorname{Tr}$ and $2 / 5 \mathrm{Tr}$ as the streamlines show. The mainstream ingestion occurs along the rotating cavity wall while the purge flow egestion appears along the stationary cavity wall, thus the anticlockwise vortices are developed in the inclined part of the cavity. The ingress is significant at the time instances of $3 / 5 \mathrm{Tr}$ and $4 / 5 \mathrm{Tr}$ as the ingress of the mainstream has passed the inclined part of cavity and reached the perpendicular part of the cavity. The notable negative axial vorticity A occupies the inclined part of the cavity. 
At cutting plane 11, the egress appears at all these five time-instances as shown by the streamlines. As a result, the negative axial vorticity $\mathrm{C}$ near the rotating wall is pumped into the mainstream and the positive axial vorticity B near the stationary hub is pushed radially, in particular at $1 / 5 \mathrm{Tr}$, where the vorticity labels are marked in the right corner view of the Fig.10. As the rotor rotates, the radial locations of positive axial vorticity $B$ and negative axial vorticity $\mathrm{C}$ gradually reduce. At $4 / 5 \mathrm{Tr}$, the positive axial vorticity $\mathrm{B}$ nearly covers the cavity exit and the negative axial vorticity $\mathrm{C}$ nearly disappears. From the time-averaged results, at cutting plane 7 , obvious anti-clockwise vortices and corresponding negative axial vorticity $A$ are formed near the cavity exit due to the ingress of the mainstream. At cutting plane 11, relative weak negative axial vorticity $\mathrm{C}$ is generated near the rotating hub and the positive axial vorticity B near the stationary hub is pushed radially by the egress of the purge flow.

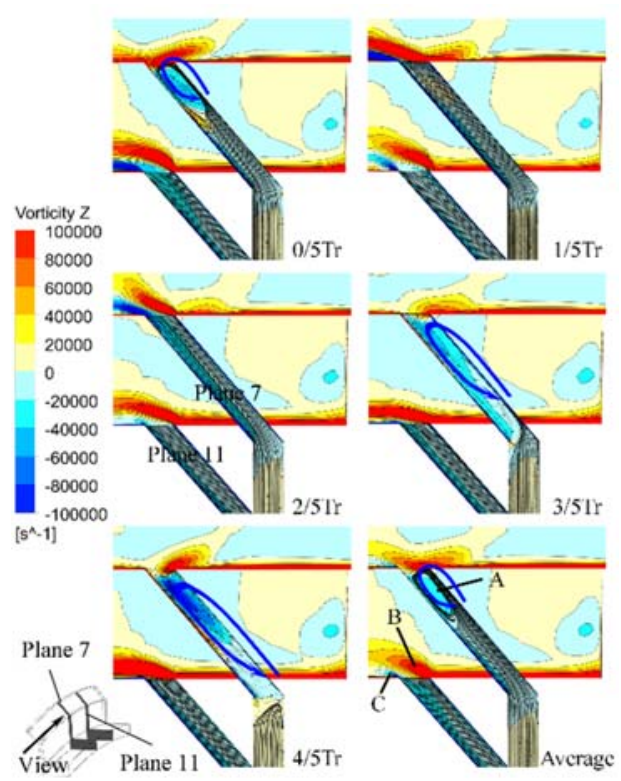

Fig. 9. Axial vorticity distributions for IR = $0.5 \%$.

To further give an insight into the unsteady feature of axial vorticity distributions related to the ingress and egress, time-resolved radial velocity and axial vorticity contours at the cavity exit for IR $=0.5 \%$ are presented Fig. 10 and Fig. 11, respectively. The unsteady ingress and egress distributions downstream of the first stator are labeled as line a and $b$. High pressure regions are located at the zone between stator trailing edge and rotor leading edge. However, low pressure regions are occurred downstream of the stator suction side and upstream of the rotor suction side. With the rotation of the rotor, when the stator trailing edge is passed by the rotor leading edge, the ingress becomes substantial at $4 / 5 \mathrm{Tr}$ between location 6 and 8 . Whereas, at $1 / 5 \mathrm{Tr}$, the rotor suction side passing by the stator suction side leads to the most significant egress between location 10 and 13. Therefore, the circumferential locations of ingress and egress change periodically as the rotor rotates. The unsteady distributions of ingress and egress at cutting planes 7 and 11 are well consistent to the streamline distributions in Fig. 9.

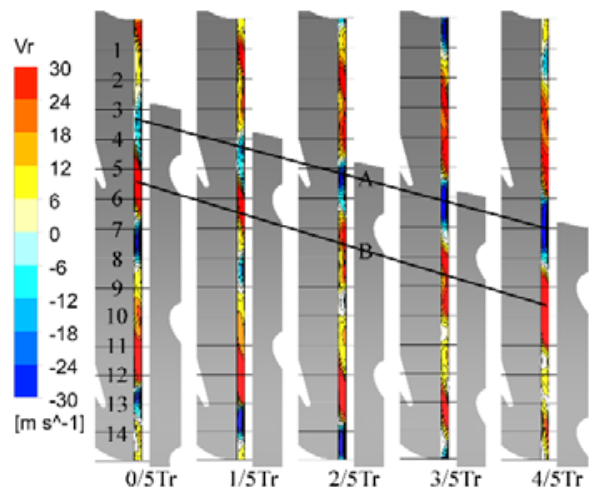

Fig. 10. Time-resolved radial velocity at cavity exit for IR $=0.5 \%$.

The circumferential locations of positive and negative axial vorticity also change periodically as the rotor rotates. Comparing the unsteady distributions of radial velocity with that of axial vorticity, it can be seen that the ingress corresponds to the positive axial vorticity while most of the egress regions are covered by the negative axial vorticity. However, some positive axial vorticity occurs in the circumferential regions of egress, labeled as square $\mathrm{D}, \mathrm{E}$ and $\mathrm{F}$. This is because the high circumferential velocity ingress of the mainstream is blocked by the purge flow inside the cavity and ejected into the main passage at other circumferential regions along the rotating direction, as shown the label $\mathrm{D}, \mathrm{E}$ and $\mathrm{F}$ in Fig. 11.

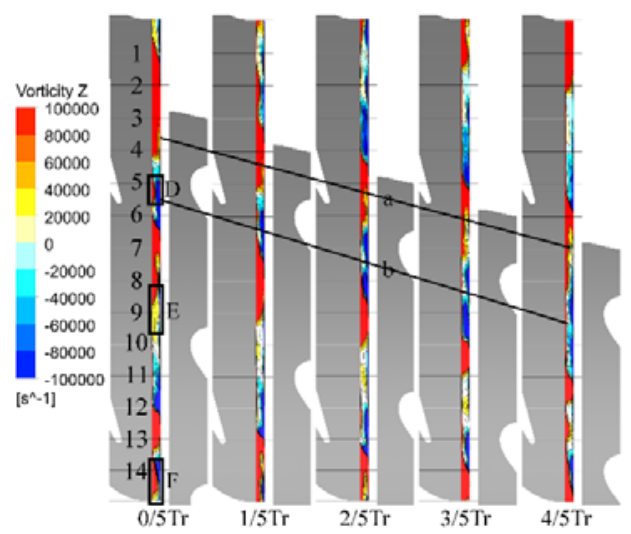

Fig. 11. Time-resolved axial vorticity at cavity exit for IR $=\mathbf{0 . 5 \%}$.

The surface streamline of axial plane at NAL $=0.50$ inside the cavity at $0 / 5 \mathrm{Tr}$ is shown in Fig. 12. The mainstream ingestion occurs between location 3 and 4 and ejected into the main passage near location 5 , as shown by label $\mathrm{D}$. Thus, there exists positive axial 
vorticity near location 5 in region $\mathrm{D}$. The positive axial vorticity in regions $\mathrm{E}$ and $\mathrm{F}$ are originated from the ingress vorticity between location 7 and 8 and between location 12 and 13, respectively. In addition, the size and intensity of ejecting positive axial vorticity is lower than that of ingress positive axial vorticity. This implies the purge flow interacts with the ingress of the mainstream, which decreases the intensity of ingress positive axial vorticity.

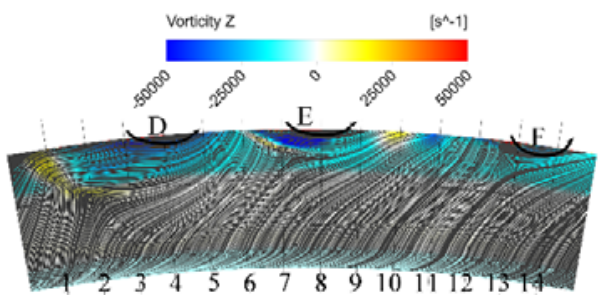

Fig. 12. Surface streamline at NAL $=0.50$ for $I R$ $=0.5 \%$ at $0 \mathrm{Tr}$.

To further investigate the vorticity change associated to the egress, the axial vorticity and circumferential velocity contours at $1 / 5 \mathrm{Tr}$ for $\mathrm{IR}=1.3 \%$ are given in Fig. 13. The egress at $1 / 5 \mathrm{Tr}$ is the most significant at plane 11 . Streamlines from the hub to $6 \%$ span are displayed to investigate axial vorticity change associated with the egress flow.

The positive axial vorticity $\mathrm{B}$ near the rotating wall is pumped into the mainstream and the negative axial vorticity $\mathrm{C}$ near the stationary hub is pushed radially at cutting planes 7 and 11 . At plane 11, an anticlockwise vortex is formed near the stator domain exit and below the negative axial vorticity $\mathrm{C}$, as labeled by the blue arrow. This vortex is developed owing to the interaction between the egress flow and mainstream near the rotating hub. Furthermore, additional anti-clockwise vortex (labeled by the blue arrow) is formed upstream of the positive axial vorticity $\mathrm{B}$ as the strong egestion blocks the main flow, then the positive axial vorticity region is decreased.

Compared with the axial vorticity at 1/5Tr in Fig. 9, both positive axial vorticity $\mathrm{B}$ and negative axial vorticity $\mathrm{C}$ are more significant for $\mathrm{IR}=1.3 \%$ than $\mathrm{IR}=0.5 \%$. More purge flow is rotated and sheared by the relative rotation of cavity walls with the increasing IR and the negative axial vorticity close to the rotating wall inside the cavity strengthens. Therefore, the corresponding negative axial vorticity $\mathrm{C}$ near the rotating hub is intensified.

In Fig. 13 (b), circumferential velocity gradients are formed at most of radial positions, labelling by the black arrow. The reason is that circumferential velocity of rim seal purge flow is low, and circumferential velocity of the mainstream is high, then the purge flow egestion impacts on the mainstream, resulting in the radial circumferentialvelocity gradient, which are consistent with positive axial vorticity $B$, shown in Fig. 13 (a). This is because the axial vorticity is just defined by radial circumferential-velocity gradients and circumferential radial-velocity gradients. The circumferential velocity difference between the mainstream and purge flow dominates velocity difference for three directions, and radial circumferential-velocity gradient develops into the positive axial vorticity. From IR $=0.5 \%$ to $\mathrm{IR}=$ $1.3 \%$, the velocity of the purge flow increases and the corresponding time passing through the cavity decreases. Then, the accelerating effect on the purge flow from the rotating wall in the circumferential direction is reduced. Thus, the radial circumferentialvelocity gradient and corresponding positive axial vorticity B strengthen with the increasing IR.

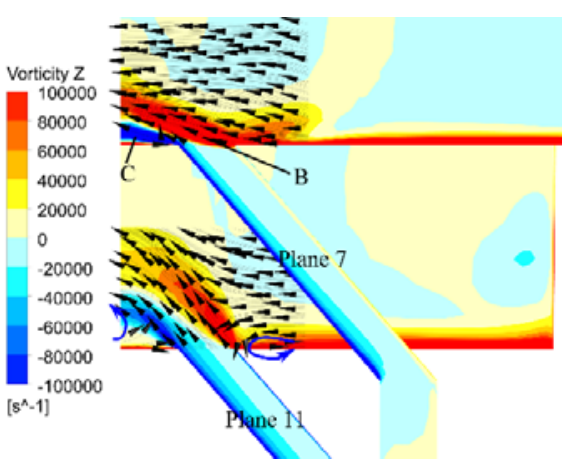

(a) Axial vorticity

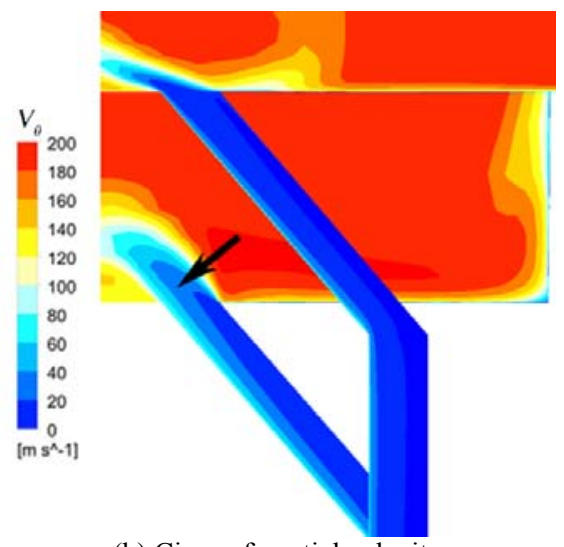

(b) Circumferential velocity

Fig. 13. Axial vorticity and circumferential velocity distributions for $I R=1.3 \%$ at $1 / 5 T$.

To summarize, inside the cavity, the vortex is generated owing to the combination effects of cavity walls rotation and non-uniform circumferential pressure caused by the upstream stator. The negative axial vorticity resulting from the relative rotation of cavity walls dominates inside the cavity and mainly situates near the rotating wall. In addition, significant ingress occurs under IR of $0.5 \%$, which produces obvious negative axial vorticity in the inclined part of the cavity. The purge flow interacts with ingress of the mainstream, leading to the intensity of the positive axial vorticity for ingress of the mainstream to decrease. This ingress of the mainstream is ejected into the main passage along the rotating direction. Furthermore, the egress flow carries the negative axial vorticity and ejects into the main flow near the 
rotating hub. The effect of the purge flow on the mainstream, especially the circumferential velocity difference, results in significant positive axial vorticity. The strong egress flow has significant blockage effect on the mainstream upstream of the cavity exit, which induces anti-clockwise vortices near the stationary hub. Furthermore, the influence of the strong egress flow on the mains flow near the rotating hub causes anti-clockwise vortices near the stator domain exit.

\subsection{Downstream of the cavity exit}

The axial vorticity distributions downstream of the cavity exit are given in Fig. 14. As compared to distributions at stator exit in Fig. 6, the size and intensity of HPV and HTSV reduce with the dissipation effect in the transport process. Their locations move to the middle span slightly with the extrusion of egress flow. Additionally, the area of positive vorticity 1 gradually increases with the increasing IR, in particular downstream of the stator suction side. This is mainly because the circumferential velocity difference between the egestion flow and mainstream strengthens with the increment of purge flow rate along radial direction, which causes intensifying positive axial vorticity. In addition, negative axial vorticity 2 exists below the positive axial vorticity from IR $=0.5 \%$ to $I R=1.3 \%$, which is in accordance with the results in Fig. 4. The negative axial vorticity is mainly formed in the cavity due to cavity walls rotation and ejected into the main passage near the rotating hub as presented in Fig. 10. The positive axial vorticity develops into the shear induced vortex and the negative axial vorticity becomes the slot leakage vortex. These two vortices are in accordance with the results of Schuepbach et al. (2011) and Jia and Liu (2013).

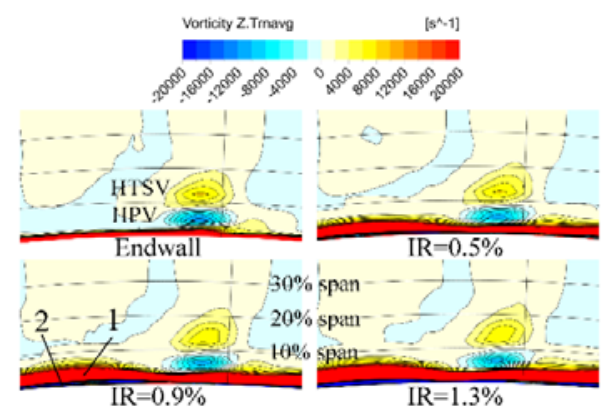

Fig. 14. Axial vorticity contours downstream of the cavity exit.

The circumferentially mass-averaged turbulence kinetic energy coefficients for three locations are given in Fig. 15. The turbulence kinetic energy coefficient is obtained by the root of turbulence kinetic energy non-dimensioned by the tip speed of the rotor. The three locations are labeled in Fig. 5. At stator exit, the decreasing intensity of HPV and HTSV as shown in Fig. 6 results in the reduction of turbulence kinetic energy coefficient between the 3\% span to $11 \%$ span. In this region, the radial location of peak values increases gradually, as shown by the red arrow. Above the cavity exit, the turbulence kinetic energy coefficients below $6 \%$ span change dramatically owing to the intrusion of the egress flow. When IR increases, the turbulence kinetic energy improves due to the positive and negative axial vorticity associated with the egress flow. Downstream of the cavity exit, the influence region of egress flow stretches to $9 \%$ span.

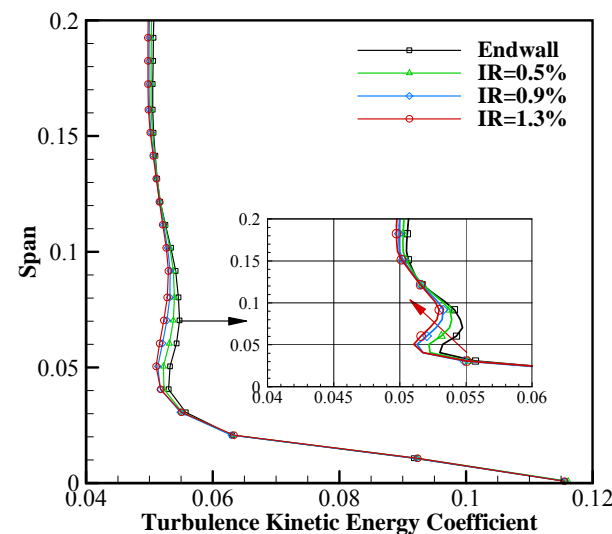

(a) At the stator exit

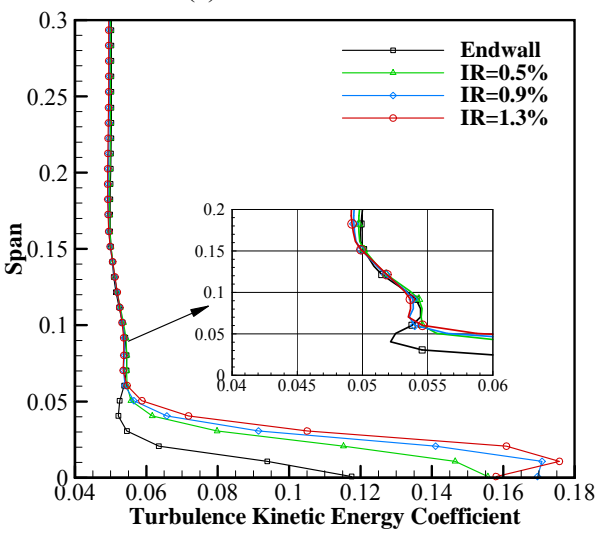

(b) Above the cavity exit

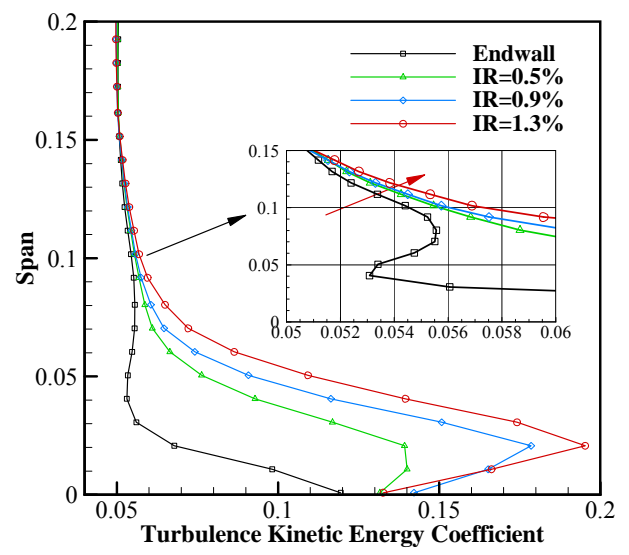

(c) Downstream of the cavity exit

Fig. 15. Distributions of turbulence kinetic energy coefficients along radial direction. 
From $\mathrm{IR}=0.5 \%$ to $\mathrm{IR}=1.3 \%$, the turbulence kinetic energy continues to strengthen and the radial locations of peak values gradually increase. Furthermore, the turbulence kinetic energy for the HPV and HTSV increase between $9 \%$ span to $15 \%$ span, as shown by the red arrow. This trend is opposite to the distribution at stator exit, which indicates that the egress flow weakens the reduction in the intensity of the HPV and HTSV in the transport process. This is because the extrusion to HPV and HTSV thins their vortex tubes and increases corresponding axial vorticity. Thus, the turbulence kinetic energy for the HPV and HTSV reduces slowly at high purge flow rate.

\section{Conclusions}

The unsteady vortex interaction between the rim seal purge flow and upstream stator has been numerically investigated. The vortex interaction processes were analyzed. In addition, the axial vorticity changes resulting from the ingress and egress were studied. The conclusions are as follows:

1) With the increment of IR, the intensity and size of HPV and HTSV at stator exit reduces, mainly due to the decreasing cross-passage gradient caused by enhancing blockage effects to the stator suction side. The regions of positive and negative axial vorticity downstream of stator trailing edge is less than that downstream of stator suction side due to the existence of a high-pressure region. From the stator exit to downstream of cavity exit, the extrusion of egress flow to the HPV and HTSV enforces the radial migration of these two vortices.

2) Inside the cavity, the negative axial vorticity is mainly induced by the combination effects of relative rotation of cavity walls and non-uniform circumferential pressure imposed by the upstream stator. The ingress causes the positive axial vorticity near the stator hub to ingest into the cavity. The purge flow interacts with the ingress of the mainstream, leading to the decrease in the intensity of positive axial vorticity. Furthermore, the interaction between the ingress of the mainstream and purge flow induces obvious anti-clockwise vortex and corresponding negative axial vorticity in the inclined part of the cavity. In addition, the ingress of the mainstream along the rotating direction due to the blockage of the purge flow in the cavity.

3) At the cavity exit, the interaction between the egress flow and the mainstream, especially the circumferential velocity difference, results in significant positive axial vorticity. The egress flow carries negative axial vorticity formed inside the cavity and enters into the main passage near the rotating hub. The strong egress flow significantly blocks the mainstream upstream of the cavity exit, which induces anti-clockwise vortices near the stationary hub. Furthermore, the interaction between the strong egress flow and mainstream near the rotating hub causes anti-clockwise vortices near the stator domain exit. The circumferential velocity differences between the egress flow and mainstream results in significant positive axial vorticity in the main passage and strengthens with the increasing IR. Owing to the relative motion of the rotor and stator, the vorticity changes caused by the ingress and egress are unsteady and circumferentially nonuniform.

4) Downstream of the cavity exit, the size and intensity of HPV and HTSV reduce with the dissipation effect in the transport process. Their locations move to the mid-span slightly with the extrusion of egress flow. The turbulence kinetic energy for the HPV and HTSV increase between 9\% span to $15 \%$ span. This trend is opposite to the distribution at stator exit, which indicates that the egress flow weakens the reduction in the intensity of the HPV and HTSV in the transport process. This is because the extrusion to HPV and HTSV thins their vortex tubes and increases corresponding axial vorticity.

\section{ACKNOWLEDGEMENTS}

The authors would like to express their gratitude for support of National Natural Science Foundation of China (No. 51876176), Natural Science Foundation of Shaanxi Province (No. 2021JC-10) and National Science and Technology Major Project (J2019- II -0015-0036).

\section{REFERENCES}

Behr, T. (2007). Control of Rotor Tip Leakage and Secondary Flow by Casing Air Injection in Unshrouded Axial Turbines. Ph. D. thesis, Dresden University of Technology, Dresden, Germany.

Blanco, E. D. and H. P. Hodson (2006, May). Effect of the Leakage Flows and the Upstream Platform Geometry on the Endwall Flows of a Turbine Cascade. In Proceedings of ASME Turbo Expo 2006: Power for Land, Sea, and Air, Barcelona, Spain, ASME 2006-GT90767.

Chilla, M., H. Hodson, and D. Newman (2012, June). Unsteady Interaction between Annulus and Turbine Rim Seal Flows. In Proceedings of ASME Turbo Expo 2012: Turbine Technical Conference and Exposition, Copenhagen, Denmark, ASME 2012-GT-69089.

Cui, J. and P. Tucker (2016, June). Numerical Study of Purge Flows in a Low-Pressure Turbine. In Proceedings of ASME Turbo Expo 2016: Turbomachinery Technical Conference and Exposition, Seoul, South Korea, ASME 2016GT-56789.

Gallier, K. D. and P. B. Lawless (2004, June). Development of the Unsteady Flow on a Turbine Rotor Platform Downstream of a Rim Seal. In Proceedings of ASME Turbo Expo 2006: Power for Land, Sea, and Air, Vienna, Austria, ASME 2004-GT-53899.

Gao, Z., D. P. Narzary, S. Mhetras and J. C. Han (2007). Upstream Vortex Effect on Turbine 
Platform Film Cooling with Typical Purge Flow. Journal of Thermophysics and Heat Transfer 26(11), 75-84.

Gibson, J. (2011). Influence of Rim Seal Purge Flow on Performance of an Endwall Profiled Axial Turbine Geometric Effects on Static Turbine Rim Seals. PhD thesis, Pennsylvania State University.

Gier, J., B. Stubert, B. Brouillet and L. Vito (2003, June). Interaction of Shroud Leakage Flow and Main Flow in a Three-Stage LP Turbine. In Proceedings of ASME Turbo Expo 2003, collocated with the 2003 International Joint Power Generation Conference, Atlanta, Georgia, USA, ASME 2003-GT-36894.

Hu, J. L., Q. Du, J. Liu P. Wang, G. Liu, H. R. Liu and M. M. Du (2017). Flow Development through HP \& LP Turbines, Part II: Effects of the Hub Endwall Secondary Sealing Air Flow on the Turbine's Main Flow. Journal of Thermal Science 26(4), 308-315.

Jia, W. and H. X. Liu (2013). Numerical Investigation of the Interaction between Upstream Cavity Purge Flow and Mainstream in Low Aspect Ratio Turbine Cascade. Chinese Journal of Aeronautics 26(1), 85-93.

Jia, W. and H. X. Liu (2014, June). Numerical Investigation of the Interaction between Upstream Purge Flow and Mainstream in a Highly-loaded Turbine. In Proceedings of ASME Turbo Expo 2014: Turbomachinery Technical Conference and Exposition, Dusseldorf, Germany, ASME 2014-GT25501.

Jia, X. Y., H. Zhang and Y. T. Jiang (2018). Performance of Radial-axial Clearance Rim Seal in Realistic Working Conditions. Aerospace Science and Technology 77(3), 373-387.

Popović, I. and H. P. Hodson (2010, June). Aerothermal Impact of the Interaction between Hub Leakage and Mainstreams in Highlyloaded HP Turbine Blades. In Proceedings of ASME Turbo Expo 2010: Power for Land, Sea, and Air, Glasgow, UK, ASME 2010-GT22311.

Schadler, R., A. I. Kalfas and R. S. Abhari (2016, June). Modulation and Radial Migration for
Turbine Hub Modes by the Rim Seal Purge Flow. In Proceedings of ASME Turbo Expo 2016: Turbomachinery Technical Conference and Exposition, Seoul, South Korea, ASME 2016-GT-56661.

Schlienger, J., A. Pfau, A. I. Kalfas and R. S. Abhari (2003, June). Effect of the Labyrinth Seal Varation on the Multistage Axial Turbine Flow. In Proceedings of ASME Turbo Expo 2003, collocated with the 2003 International Joint Power Generation Conference, Atlanta, Georgia, USA, ASME 2003-GT-38270.

Schobeiri, M. T., K. Lu and M. Rezasoltani (2015). Effect of Non-axisymmetric Contouring on Performance and Film Cooling of a Rotating Turbine Endwall Subjected to the Secondary Air Purge: A Combined Numerical and Experimental Study. Proceeding of IMechE Part A: Journal Power and Energy 229(8), 813-831.

Schuepbach, P., R. S. Abhari, M. G. Rose and J. Gier (2011). Influence of Rim Seal Purge Flow on the Performance of an Endwall-Profiled Axial Turbine. Journal of Turbomachinery 133, 021011-021011-10.

Schuepbach, P. (2009). Influence of Rim Seal Purge Flow on Performance of an Endwall Profiled Axial Turbine. Ph. D. thesis, Swiss Federal Institute of Technology, Swiss.

Turgut, O. H. and C. Camc1 (2012, June). Computational Validation of the Flow through a Turbine Stage and the Effects of Rim Seal Cavity Leakage on Secondary Flows. In Proceedings of ASME Turbo Expo 2012: Turbine Technical Conference and Exposition, Copenhagen, Denmark, ASME 2012-GT69306.

Wright, L. M., S. A. Blake, D. H. Rhee and J. C. Han (2007, May). Effect of Upstream Wake with Vortex on Turbine Blade Platform Film Cooling with Simulated Stator-Rotor Purge Flow. In Proceedings of ASME Turbo Expo 2007: Power for Land, Sea, and Air, Montreal, Canada, ASME 2007-GT-27092.

Zhu, L. Y., X. Luo, G. Q. Xu, et al. (2014). A Theoretical and Numerical Study of Turbine Rim Seal Ingestion. Journal of Propulsion Technology 35(11), 1511-1519. 